\title{
Verlauf von aggressivem Picken und einigen Verhaltensmerkma- len in rangordnungsinstabilen Käfiggruppen bei Legehennen (Kurzmitteilung)
}

\begin{abstract}
Title of the paper: Progress of aggressive pecks and several behavioral traits in rank-order-instable cage groups in laying hens (short communication)

Higher animals establish a rank-order, which provides advantages to the social life of these animals. The rankorder moderates aggression and decreases stress-based frustration. Laying hens kept in cages are normally replaced with new ones after they die. This results in conflict in the rank-order of the animals, particularly in adult hens depending on breed and line and, as a result, causes violation among animals. This is a stress situation, which can negatively affect welfare of the animals. In this study, the effect of instable rank-order on aggressive pecking and some behavioral traits was investigated in laying hens. Fourty eight white Lohmann LSL laying hens were used as animal material. The stocking rate was 4 laying hens in all cages, except for six cages in which a stable rank-order was formed with animals who came from different cages. The behavioral traits of aggressive pecking, feeding, drinking, feather pecking, cage pecking, standing and recumbency were monitored. A significant difference in aggressive pecking was found between stable rank-order and instable rank-order groups. The laying hens in instable-rank-order group showed more frequently aggressive pecking than those in rank-order-stable group. However, the progress of aggressive pecking during the experimental period indicated an elevation of aggressive pecking in both of the groups. The reason for increased aggressive pecking in the rank-order-stable group was thought to be due to the threatening with noise of the animals in the rank-orderinstable group. Feather pecking was significantly higher in the instable rank-order group than in the stable rankorder group, indicating the frustration of the hens in the former group. Feather pecking is normally defined as a comfort behavior, but is not observed in every situation. This behavior could be classified as a "sparking over activity".
\end{abstract}

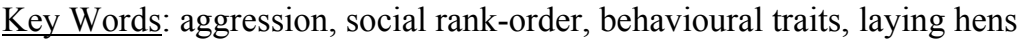

\section{Zusammenfassung}

Die hochentwickelten sozialen Tiere bilden untereinander eine Rangordnung, die für das soziale Leben dieser Tiere Vorteile bringt. Die Rangordnung hemmt die Agression und mildert stressbedeutende Frustration. Bei der Käfighaltung der Legehennen werden die Käfige, wegen Mortalität zeitweise neu besetzt. Dieses führt zu den Rangordnungskämpfen, die bei adulten Hennen, je nach Rasse und Linie, heftig verlaufen können. Es ist eine Stresssituation, die das Wohlbefinden der Tiere negativ beeinträchtigt. In dieser Arbeit wurden der Einfluss der instabilen Rangordnung von Käfiggruppen auf das aggressive Picken und einige Verhaltensmerkmale bei Legehennen untersucht. 48 weisse Lohmann LSL Legehennen dienten als Tiermaterial. Die Besatzdichte war vier Legehennen pro Käfig, wobei in sechs Käfigen eine stabile Rangordnung war. Zusätzlich wurden in sechs Käfigen Tiere aus unterschiedlichen Käfigen zusammengestellt. Es wurden die Verhaltensmerkmale aggressives Picken, Fressen, Trinken, Federputzen, Käfigpicken, Federpicken, Stehen und Liegen beobachtet. Zwischen der Instabilen- und Stabilenrangordnungsgruppe wurde ein signifikanter Unterschied für das aggressive Picken beobachtet. Die Hennen in den rangordnungsinstabilen Gruppen zeigten das aggressive Picken häufiger als die Hennen in den rangordnungsstabilen Gruppen. Allerdings deutete der Verlauf des agressiven Pickens während der Versuchsperiode auf eine Erhöhung des aggressiven Pickens in beiden Gruppen hin. Als Ursache für das erhöhte aggressive Picken bei den rangordnungsstabilen Gruppen wurden die Drohlaute der Hennen in den rangordnungsinstabilen Gruppen interpretiert. Auch das Federputzen war bei den rangordnungsinstabilen Gruppen signifikant höher als bei den rangordnungsstabilen Gruppen, was auf eine deutliche Frustration der Hennen in den rangordnungsinstabilen Gruppen deutet. Das Federputzen wird zwar als ein Komfortverhalten definiert, was 
jedoch nicht in jeder Situation gilt. In der vorliegenden Studie kann man es als Übersprungsbewegung definieren.

Schlüsselwörter: Aggression, soziale Rangordnung, Verhaltensmerkmale, Legehennen

1 . Einleitung

Die weitverbreitetste Betriebsform der kommerziellen Eierproduktion ist die Käfighaltung. In der Regel werden die einzelnen Käfige mit vier oder fünf Hennen besetzt. Kurz nach der Aufstallung der Junghennen wird in den einzelnen Käfiggruppen eine relativ stabile Rangordnung gebildet. Nach eigenen Beobachtungen sind die Rangordnungskämpfe der Jungtiere nicht so heftig, währenddessen die Kämpfe der adulten Tiere physische Schäden hinzufügend verlaufen können.

Besonders bei Ressourcenmangel, wie z.B. Futter, Lebensraum sowie Geschlechtspartner, hat die Aggression eine lebenserhaltende Funktion (HOUPT, 1998). Die Aggression wird bei den Legehennen von mehreren Faktoren, wie z.B. Genotyp, Alter, Gruppengrösse, Lichtstärke und -länge beeinflusst (NICOL et al., 1999; McADIE und KEELING, 2000; HUBER-EICHER und SEBÖ, 2001; KJAER und VESTERGAARD, 1999). Es ist z.B. bekannt, dass die weissen Legehybriden aggressiver sind als die braunen Legehybriden (SAVAŞ und ŞAMLI, 2000).

Die Mortalität während der Produktionsphase führt zur Unausgeglichenheit der Käfigbesetzungen, worauf die Käfiggruppen mehrmals ausgeglichen werden können. Das wiederum führt zur Instabilisierung der sozialen Rangordnung bei diesen Käfigen. Diese Studie befasst sich mit dem Einfluss der Rangordnungsinstabilisation in Käfiggruppen bei adulten Legehennen auf einige Verhaltensmerkmale, wobei auf die Aggression ein besonderes Augenmerk gerichtet wird.

\section{$2 . \quad$ Material und Methoden}

Als Tiermaterial dienten 48 weisse Lohmann LSL Legehennen, die in der zweiten Produktionsphase waren. Die Besatzdichte war vier Legehennen pro Käfig, wobei in sechs Käfigen Tiere waren, die eine stabile Rangordnung hatten. Zusätzlich wurden in sechs Käfigen, Tiere aus unterschiedlichen Käfigen zusammengestellt. Das Management der Tiere unterlag einem Standardverfahren für die Legehennen in der Käfighaltung.

Es wurden die folgenden Verhaltensmerkmale beobachtet: aggressives Picken, Fressen, Trinken, Federputzen, Käfigpicken, Federpicken, Stehen und Liegen.

Die Tiere wurden 10 Tage lang dreimal am Tag jeweils 15 Minuten zwischen $9^{00}-9^{15}$, $12^{\underline{00}}-12^{\frac{15}{5}}$ und $15^{\frac{00}{\underline{0}}}-15^{\frac{15}{5}}$ Uhr gefilmt. Die totale Video-Aufnahmezeit betrug 7,5 Stunden. Ausser des aggressiven Pickens wurden die Aufnahmen mit der TimeSampling Methode ausgewertet, wobei die Anzahl der Hennen pro Käfig, die das Verhalten ausüben, in einminütigen Abständen eingetragen wurden (BOGNER, 1984). Beim aggressiven Picken hingegen wurde die Anzahl der beobachteten Tiere pro Käfig während der ganzen Beobachtungsphase (15 Minuten) registriert. Die statistische Auswertung der Daten wurde mit dem Programmpaket SAS (1996) ausgeführt. Dabei wurde für das aggressive Picken ein lineares Modell mit den fixen Effekten Rangordnungsverhältnisgruppe, Beobachtungstag und Beobachtungsperiode angewandt. Die übrigen Verhaltensmerkmale wurden mit einem Schwellenwertmodell und den fixen Effekten Rangordnungsverhältnisgruppe, Beobachtungstag und Beobachtungsperiode 
analysiert. Zur Beurteilung der Einflussgrösse Rangordnungsverhältnis und der des Schwellenmerkmalsmodells zugrundeliegenden Verhaltensmerkmale kamen die geschätzten Regressionskoeffizienten, deren Standardfehler und die „odds ratios“ zur Anwendung (SAVAŞ et al., 2001).

\section{Ergebnisse und Diskussion}

In Tabelle 1 sind die Ergebnisse für aggressives Picken und Rangordnungsverhältnisgruppe wiedergegeben. Ein deutlich höherer signifikanter Wert ist für die instabile Rangordnungsgruppe gegenüber der stabilen Rangordnungsverhältnisgruppe festzustellen. Aus den LS-Mittelwerten des absoluten aggressiven Pickens lassen sich bei den rangordnungsinstabilen Käfiggruppen ein totaler Wert von 2,4 und für die rangordnungsstabilen Käfiggruppen ein totaler Wert von 1,4 pro Beobachtungsperiode und -tag kalkulieren. Allerdings bezieht sich das aggressive Picken bei den beiden Versuchsgruppen auf unterschiedliche Motive. Während das aggressive Picken bei den rangordnungsinstabilen Käfiggruppen zur Klärung der Rangordnung angewandt wird, dient es bei den rangordnungsstabilen Käfiggruppen wesentlich zur Vertreibung der Rangunteren.

Tabelle 1

LS-Mittelwerte $(\bar{X})$ und deren Standardfehler (SE) für die Rangordnungsverhältnisgruppen und aggressives Picken pro Henne, Käfig, Beobachtungsperiode und -tag (LSQ-means ( $\bar{X})$ and their standard errors (SE) of the rank-order groups and aggressive pecking per hen-cage-observation period-observation day)

\begin{tabular}{cccc}
\hline \multicolumn{2}{c}{ Rangordnungsverhältnisgruppe } & & $\mathrm{P}<$ \\
Stabil & Instabil & SEM & 0,001 \\
0,06 & 0,10 & 0,01 & \\
\hline
\end{tabular}

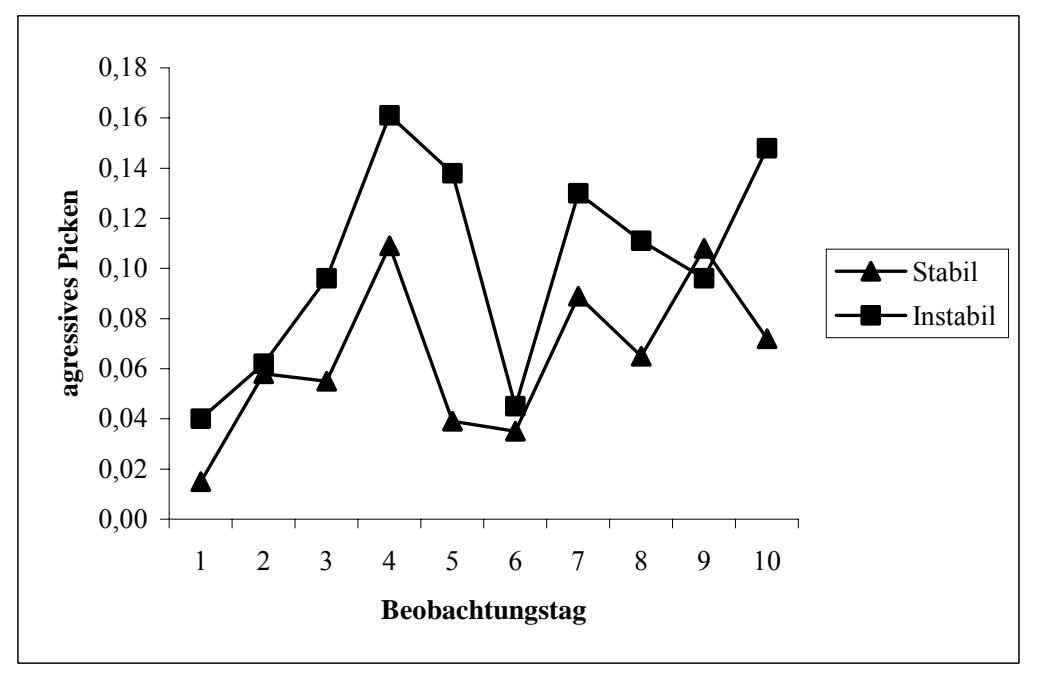

Abb.: Verlauf des aggressiven Pickens pro Henne, Käfig, Beobachtungsperiode und -tag in rangordnungsstabilen und rangordnungsinstabilen Gruppen (Progress of aggressive pecking per hen-cage-observation periodobservation day in stable and instable rank-order groups)

Die Abbildung zeigt die Verläufe des aggressiven Pickens für die beiden Versuchsgruppen während des Versuchszeitraumes. Das aggressive Picken zeigt erst am vierten Versuchstag einen Peak. Es scheint, dass die ersten Tage eher mit der Erkundung der neuen Käfigumgebung verbracht wurden und nicht mit den Rangordnungskämpfen. In den ersten Tagen sind die Tiere mit der Umgebung noch nicht vertraut, um sich mit den anderen Hennen auseinander zu setzen. Der Peak am vierten Tag reicht wahr- 
scheinlich nicht aus, um die Rangordnungsverhältnisse zu klären. Nach heftigen Auseinandersetzungen am vierten und fünften Tag machen sie eine Pause, die wahrscheinlich zur Bedarfsdeckung anderer Lebensfunktionen dient. Wahrscheinlich versuchen die unterlegenen Hennen immer wieder sich durchzusetzen und einen höheren Rang zu erreichen. Andererseits ist auch die Aggressivität der ranghöheren Hennen gegenüber den rangunteren Hennen noch hoch, die eigentlich bei miteinander vertrauten Individuen sinkt.

Interessanterweisse zeigt das aggressive Picken auch bei der rangordnungsstabilen Gruppe einen ähnlichen Verlauf, wenn auch auf einem niedrigeren Niveau. Das deutet auf eine Stimulation der rangordnungsstabilen Gruppen durch die rangordnungsinstabilen Gruppen hin. Die Tiere sahen sich nicht, jedoch konnten sie sich hören. Die Drohlaute der Hennen in den rangordnungsinstabilen Gruppen induktieren wahrscheinlich die Aggression bei den ranghöheren Hennen in den rangordnungsstabilen Gruppen. Daher spiegelt der Mittelwert des aggressiven Pickens für die rangordnungsstabile Gruppe nicht den normalen Wert wider (Tab. 1).

In der Tabelle 2 sind die Ergebnisse der $\mathrm{Chi}^{2}$-Werte und die Signifikanzangaben der Einflussgrößen auf die beobachteten Verhaltensmerkmale zusammengefasst. Das Verhalten Fressen zeigt in beiden Käfiggruppen signifikant unterschiedliche Häufigkeiten. Hingegen ist der Unterschied der Käfiggruppen für das Trinken nicht signifikant. Ein deutlich signifikanter Einfluss der Rangordnungsverhältnisgruppen ist für das Federputzen zu beobachten. Die gleiche Einflussgröße hingegen ist für die Verhalten Käfigpicken und Federpicken nicht signifikant. Das Liegen und das Stehen zeigen signifikante Unterschiede zwischen den beiden Rangordnungsverhältnisgruppen. Der Einfluss der Einflussgrößen Beobachtungstag und -periode sind für alle beobachteten Verhaltensmerkmale signifikant.

Tabelle 2

Chi'-Werte $\left(\chi^{2}\right)$ und Signifikanzangaben $(\mathrm{P})$ der Einflussgrößen auf die beobachteten Verhaltensmerkmale (Chi-square values $\left(\chi^{2}\right)$ and significance statement $(\mathrm{P})$ of main factors in relation to the observed behaviour traits)

\begin{tabular}{lcccccc}
\hline \multirow{2}{*}{ Merkmal } & \multicolumn{2}{c}{ Rangordnungsverhältnis } & \multicolumn{2}{c}{ Beobachtungstag } & \multicolumn{2}{c}{ Beobachtungsperiode } \\
\cline { 2 - 7 } & $\chi^{2}$ & $\mathrm{P}$ & $\chi^{2}$ & $\mathrm{P}$ & $\chi^{2}$ & $\mathrm{P}$ \\
\hline Fressen & 3,97 & $*$ & 85,24 & $* *$ & 31,20 & $* *$ \\
Trinken & 0,37 & N.S. & 48,41 & $* *$ & 8,61 & $*$ \\
Federputzen & 9,49 & $* *$ & 174,29 & $* *$ & 41,06 & $* *$ \\
Käfigpicken & 0,93 & N.S. & 37,78 & $* *$ & 15,31 & $* *$ \\
Federpicken & 2,47 & N.S. & 32,03 & $* *$ & 6,71 & $*$ \\
Liegen & 5,49 & $*$ & 326,82 & $* *$ & 126,53 & $* *$ \\
Stehen & 6,22 & $*$ & 183,93 & $* *$ & 8,22 & $*$ \\
\hline
\end{tabular}

N.S. nicht signifikant; ${ }^{*} \mathrm{P}<0,05 ;{ }^{* *} \mathrm{P}<0,01$

Die für den Vergleich der rangordnungsstabilen und -instabilen Käfiggruppen angewandten Parameterwerte sind in der Tabelle 3 zu sehen. Die Hennen der rangordnungsinstabilen Gruppen fressen $0,4 \%(\Psi=1,04)$ häufiger als die Hennen der rangordnungsstabilen Gruppen. Dieser Wert mag zu klein erscheinen. Jedoch wenn man beachtet, dass das Merkmal Fressen das am häufigsten ausgeführte Verhalten ist, ist es nicht erstaunlich, dass diese Differenz schon zum signifikanten Unterschied zwischen den beiden Gruppen führt. Es scheint, dass die häufigeren Auseinandersetzungen der Hennen in den instabilen Gruppen mehr Energie verzehren und daher diese 
Hennen häufiger fressen. Unbedeutend ist hingegen die $0,3 \%$-ige Differenz ( $\Psi=$ 0,97) der Gruppen beim Trinken (Tab. 2).

\section{Tabelle 3}

Regressionskoeffizienten (b), deren Standardfehler (SE) und odds ratios $(\Psi)$ der Einflussgröße Rangordnungsverhältnis und der beobachteten Verhaltensmerkmale (Regression coefficients (b) and their standard errors (SE) and odd ratios ( $\Psi$ ) of the rank-order group as a main factor on the observed behaviour traits)

\begin{tabular}{l|c|c|c}
\hline \multirow{2}{*}{ Merkmal } & \multicolumn{2}{|c}{ Rangordnungsinstabile Käfiggruppen $^{1}$} \\
\cline { 2 - 4 } & $\mathrm{b}$ & SE & $\Psi$ \\
\hline Fressen & 0,04 & 0,021 & 1,04 \\
Trinken & $-0,03$ & 0,056 & 0,97 \\
Federputzen & 0,11 & 0,036 & 1,12 \\
Käfigpicken & $-0,06$ & 0,061 & 0,94 \\
Federpicken & $-0,31$ & 0,199 & 0,73 \\
Liegen & $-0,14$ & 0,059 & 0,87 \\
Stehen & $-0,06$ & 0,025 & 0,94 \\
1 Die Regressionskoeffizienten (b) und „odds ratios“ ( $\Psi$ ) für die rangordnungsstabilen Käfiggruppen sind in der Reihenfolge 0,00 und 1,00
\end{tabular}

Bemerkenswert ist das $12 \%(\Psi=1,12)$ häufigere Federputzen der Hennen in den rangordnungsinstabilen Käfiggruppen. Dieses Ergebniss erscheint auf den ersten Blick unlogisch, denn das Federputzen wird als ein Komfortverhalten definiert. Wenn jedoch bedacht wird, dass es sich um eine Übersprungsbewegung handeln könnte, ist es durchaus möglich. Es ist bekannt, dass Frustration bei Hühnern Übersprungsbewegungen in Form von Federputzen hervorrufen kann (BESSEI, 1983).

Das Käfig- und Federpicken sind Verhaltensmerkmale aus dem Funktionskreis Nahrungsaufnahme. Die Häufigkeit des Käfigpickens ist in der Käfighaltung nicht gering. Geringe Beschäftigungsmöglichkeiten in Folge von reizarmer Umgebung könnte dieses stereotypische Verhalten auslösen (DANTZER, 1986; WEMESFELDER, 1993). Die Ursachen und Funktion des Federpickens wurden in zahlreichen Studien bearbeitet (KJAER und VESTERGAARD, 1999; NICOL et al., 1999; Mc ADIE und KEELING, 2000; HUBER-EICHER und SEBÖ, 2001). In der vorliegenden Studie wurde das Federpicken bei den Hennen in den rangordnungsinstabilen Gruppen $27 \%(\Psi=0,73)$ weniger beobachtet. Jedoch gestattet der hohe Standardfehler für dieses Merkmal (SE $=0,199)$ keine statistische Absicherung der Differenz zwischen den beiden Gruppen.

Die Hennen in den rangordnungsinstabilen Gruppen zeigten das Verhalten Liegen 13 $\%(\Psi=0,87)$ weniger als die Hennen in den rangordnungsstabilen Gruppen. Auch das Stehen wurde bei den rangordnungsinstabilen Gruppen signifikant weniger $(6 \%, \Psi=$ $0,94)$ beobachtet als bei den rangordnungsstabilen Gruppen. Höchstwahrscheinlich ist es eine Folge der höheren Häufigkeit der Auseinandersetzungen und des Fressens der Hennen in den rangordnungsinstabilen Gruppen.

Es ist bekannt, dass die Rangordnung in der Gruppe vorteilhaft ist (WENNRICH, 1978). Wie auch in der vorliegenden Studie der Fall führt die Instabilisierung der Rangordnung zeitweise zu heftigen Auseinandersetzungen. Allerdings betont BESSEI (1989), dass in vielen Fällen die Rangordnungskämpfe nicht zu Leistungsdepressionen bei den Legehennen führen. Jedoch ist die Instabilisierung der Randordnung eine Frustrationssituation, die das Wohlbefinden der Hühner negativ beeinflusst. 
Ein interessantes Ergebnis hierzu ist die erhöhte Aggressivität der Hennen in den rangordnungsstabilen Gruppen, die aus den Drohlauten der Hennen in den rangordnungsinstabilen Käfiggruppen hervorgegangen ist.

BESSEI, W.:

\section{Literatur}

Verhaltensänderungen des Huhns bei Intensivierung des Haltungssystems. Arch. Gefügelk. 47 (1983), 8-16

BESSEI, W.:

Ethologische Aspekte der Geflügelproduktion. In: Entwicklungstendenzen in der Geflügelproduktion. Hohenheimer Arbeiten, Verlag Eugen Ulmer (1989), 75-89

BOGNER, H.:

Verhaltensbeobachtungen, Versuchsanlage und -auswertungen. In: Verhalten Landwirtschaftlicher

DANTZER, R.:

Nutztiere (Hrsg. BOGNER, H., GRAUVOGL, A.). Verlag Eugen Ulmer, Stuttgart (1984), 61-74

Behavioural, physiological and functional aspects of stereotyped behavior: a review and a reinterpretation. J. Anim. Sci. 62 (1986), 1776-1786

HOUPT, K.A.:

Domestic Animal Behaviour for Veterinarians and Animal Scientists. Third Edition, Manson Publishing Ltd., London (1998)

HUBER-EICHER, B.; SEBÖ, F.:

The prevalence of feather pecking and development in commercial flocks of laying hens. Appl. Anim. Behav. Sci. 74 (2001), 223-231

KJAER, J.B.; VESTERGAARD, K.S.:

Development of feather pecking in relation to light intensity. Appl. Anim. Behav. Sci. 62 (1999), 243254

MC ADIE, T.M.; KEELING, L.J.:

Effect of manipulating feathers of laying hens on the incidence of feather pecking and cannibalism. Appl. Anim. Behav. Sci. 68 (2000), 215-229

NICOL, C.J.; GREGORY, N.G.; KNOWLES, T.G.; PARKMAN, I.D.; WILKINS, L.J.:

Differential effects of increased stocking density, mediated by increased flock size, on feather pecking and aggression in laying hens. Appl. Anim. Behav. Sci. 65 (1999), 137- 152

SAS INSTITUTE INC. :

User's Guide Vers. 6.07, Cary, NC, USA (1992)

SAVAS, T.; ŞAMLI, H.E.:

Einfluss der Agression und Rangordnung auf Legeleistung und einige Verhaltensmerkmale bei Legehennen. (in Türkisch) Tarım Bilimleri Dergisi 6 (2000), 11-15

SAVAŞ, T.; YURTMAN, I.Y.; KARAAĞAÇ, F.; KÖYCÜ E.:

Einfluss der intensiven Gruppenhaltung und Geschlecht auf Oral-Stereotypien und einige Verhaltensmerkmale bei Mastlämmern. Arch. Tierz., Dummerstorf 44 (2001), 313-322

WEMELSFELDER, F.:

The concept of animal boredom and its relationship to stereotyped behaviour. In: Stereotypic Animal Behaviour: Fundamentals and Applications to Welfare (Eds. LAWRENCE, A.B., RUSHEN, J.), CAB International, Wallingford, UK (1993), 65-95

WENNRICH, G.:

Huhn. In: Nutztierethologie. Das Verhalten landwirtschaftlicher Nutztiere-Eine angewandte Verhaltenskunde für die Praxis. (Hrsg. H.H. SAMBRAUS), Verlag Paul Parey, Berlin-Hamburg (1978), 249274

Eingegangen: 28.03 .2003

Akzeptiert: 12.06 .2003

Author's addresses

Dr. FEVZI KARAAĞAÇ

Kadıköy Belediyesi Veteriner Polikliniği

İstanbul/Türkei

Doz. Dr. TÜRKER SAVAŞ

Çanakkale Onsekiz Mart Üniversitesi

Doz. Dr. MUSTAFA ÖZDEN

İstanbul Üniversitesi Veteriner Fakültesi

Zootekni Anabilim Dalı, Avcılar

Ziraat Fakültesi Zootekni Bölümü

Terzioğlu Kampüsü

Çanakkale/Türkei

İstanbul/Türkei

E-Mail: tsavas@comu.edu.tr 